\title{
Migrations et réfugiés au Proche-Orient et au Soudan - Introduction
}

\section{Roussel Cyril}

\section{(2) OpenEdition}

\section{Journals}

Édition électronique

URL : http://journals.openedition.org/rge/1968

DOI : $10.4000 /$ rge. 1968

ISSN : 2108-6478

Éditeur

Association des géographes de l'Est

Édition imprimée

Date de publication : 31 décembre 2009

ISSN : 0035-3213

Référence électronique

Roussel Cyril, « Migrations et réfugiés au Proche-Orient et au Soudan - Introduction », Revue Géographique de l'Est [En ligne], vol. 49 / 4 | 2009, mis en ligne le 21 octobre 2010, consulté le 08 septembre 2020. URL : http://journals.openedition.org/rge/1968 ; DOI : https://doi.org/10.4000/rge. 1968

Ce document a été généré automatiquement le 8 septembre 2020

Tous droits réservés 


\title{
Migrations et réfugiés au Proche- Orient et au Soudan - Introduction
}

\author{
Roussel Cyril
}

1 La table ronde «Les concentrations de populations déplacées et réfugiées au ProcheOrient ", tenue à Lyon le 25 mars 2009, constituait un prélude au lancement d'un programme collectif de recherche sur ce même thème, réalisé dans le cadre du GREMMO (Groupe de Recherches et d'Etudes sur la Méditerranée et le Moyen-Orient) Maison de l'Orient et de la Méditerranée - Université Lyon 2. Cette réunion a eu pour but de rassembler des chercheurs et des acteurs de l'humanitaire dans le but de réfléchir sur la thématique des concentrations de populations déplacées et réfugiées. C'est dans cette optique qu'est née l'association Ressources et Recherches Humanitaires (RH2) http://ressources-recherches-humanitaires.org/. L'aire géographique que nous avons délimitée dans un premier temps pour ce projet, comprend le Soudan, l'Egypte, la Syrie, le Liban et la Jordanie. Elle permet d'appréhender des situations variées et possède l'avantage de nous donner un recul historique: des réfugiés palestiniens aux populations du Darfour en passant par les Irakiens, nous avons plusieurs exemples de concentration de populations déplacées et réfugiées. Cette aire fait, bien sûr, partie d'un ensemble géopolitique plus vaste - le Moyen-Orient - dans lequel la problématique des concentrations de populations déplacées et réfugiées se pose avec acuité. C'est pourquoi nous resituons au préalable le débat dans ce contexte plus large qui met partout aux prises les acteurs supranationaux de l'humanitaire (grandes ONG occidentales) et les acteurs décisionnels étatiques ou issus de la société civile locale. De ces discussions, plusieurs grandes idées transversales sont apparues. Nous nous efforçons de les présenter ici.

\section{Un contexte favorable aux concentrations de populations déplacées et réfugiées}

2 La principale raison qui a conduit des universitaires et des humanitaires à se réunir autour de la question des concentrations de réfugiés reste intimement liée à la 
situation particulièrement conflictuelle que connaît le Proche et Moyen-Orient. Cette région du monde est caractérisée par un désordre géopolitique grandissant. Considérée par certaines nations comme un terrain de bataille dans leur lutte contre le terrorisme ("Global War On Terror») ou bien comme un espace stratégique pour leur alimentation en ressources énergétiques fossiles, cette région est devenue la principale zone pourvoyeuse de populations déplacées ou réfugiées dans le monde. De plus en plus, la problématique humanitaire émerge dans cette région périodiquement touchée par des conflits de forte intensité (Soudan, Liban, Palestine, Irak, Afghanistan) qui entraînent dans un premier temps son cortège d'images médiatiques et de détresses humaines mais, dans un second temps, les missions d'acteurs indépendants (ONG internationales, ONG locales...) ou supra-étatiques (agences onusiennes...) qui interviennent pour tenter de gérer l'urgence et l'après conflit. Dans ces conditions, les chercheurs en sciences sociales ont vu foisonner, sur le terrain, ces acteurs avec lesquels ils entrent en contact. Il nous est donc apparu naturel de chercher à échanger l'information et à faire circuler le savoir entre ces divers milieux. Car en effet, les temporalités d'action et les moyens d'intervention entre le chercheur et l'humanitaire se situent aux antipodes. L'humanitaire a plus l'habitude d'intervenir dans l'urgence avec toute une logistique derrière lui. Le chercheur, à l'inverse, doit s'insérer lentement et prudemment dans la société mais dispose de moins de moyens. Ainsi, l'humanitaire ne pourrait-il pas, afin de mieux gérer son intervention, bénéficier du savoir empirique du chercheur et ce dernier utiliser à son tour la foule d'informations et de données statistiques dont dispose le premier?

\section{L'action humanitaire occidentale au Proche et Moyen-Orient. Prégnance du politique entre Etat et société plurielle}

3 En fait, l'intervention humanitaire au Proche et Moyen-Orient n'est pas sans poser des problèmes particuliers liés à la nature même des Etats qui contrôlent le territoire, mais aussi à celle des sociétés qui l'occupent. Nous nous sommes ainsi interrogés sur les difficultés de l'intervention humanitaire dans certains pays du Proche et Moyen-Orient qui restent bien souvent réfractaires à l'ingérence étrangère dans ce qu'ils considèrent encore généralement comme leur chasse gardée. Prenons le cas de la Syrie, Etat très centralisé, autoritaire et nationaliste qui se méfie de l'influence extérieure et plus particulièrement occidentale. Nous rejoignons dans cette idée les propos de G. Chatelard et M. K. Doraï qui écrivent « la relation est malaisée entre des ONG humanitaires habituées à travailler dans des contextes de conflit où l'Etat est faible ou inexistant, et les autorités syriennes qui entendent exercer un contrôle strict sur les budgets et les programmes des agences humanitaires » et ainsi « contenir toute velléité de pression politique» (Chatelard \& Doraï, 2009). C'est pourquoi les ONG humanitaires occidentales doivent généralement passer par le truchement d'un organisme local pour exercer leur mission. En Syrie, les ONG étrangères ont dorénavant la possibilité de s'enregistrer auprès du Croissant rouge syrien pour mettre en œuvre des programmes d'assistance aux réfugiés irakiens. A noter que le phénomène est récent (2007) car, malgré les impératifs sécuritaires internes, bien souvent l'urgence de la situation risque de décrédibiliser le régime aux yeux de sa propre population si celui-ci s'avère impuissant à gérer l'urgence humanitaire. Et dans un même temps, le régime a 
rapidement perçu les intérêts politiques qu'il pourrait retirer d'un assouplissement de ses positions, véritable gage donné à la communauté internationale. Placé sur la sellette après son rôle présupposé dans les attentats de personnalités politiques au Liban, le régime syrien a tout intérêt à se montrer docile sur la scène internationale et bienveillant dans le traitement de la question humanitaire des populations réfugiées. Mais cette brèche dans le système cloisonné d'un tel Etat autoritaire ne doit pas faire oublier que l'action des ONG étrangères reste confinée à des secteurs délaissés par les pouvoirs publics qui gardent le contrôle politique (comme c'est le cas au Soudan, en l'Egypte). Cet exemple illustre le contexte politique difficile auquel doivent faire face les ONG au Proche et Moyen-Orient et qui tend à restreindre l'autonomie des ONG occidentales. Ces dernières demeurent bien souvent prisonnières $\mathrm{du}$ contexte sécuritaire.

4 La nature même des sociétés arabes s'avère parfois être un autre écueil ou plutôt une contrainte à l'action des ONG occidentales. Les sociétés arabes pluriconfessionnelles du Proche et Moyen-Orient disposent toutes d'une multitude d'associations caritatives locales qui fonctionnent pour beaucoup sur des logiques communautaires et des réseaux socio-confessionnels. Leurs champs d'action et d'intervention sont vastes, conséquence de la multiplicité de ces acteurs locaux. Parmi elles, certaines (les plus visibles) ont une portée politique affirmée comme Jihad el Bina liée au Hezbollah libanais; et les exemples sont légions dans d'autres pays de la région comme les réseaux caritatifs islamistes en lien avec le Hamas à Gaza ou les Frères Musulmans en Egypte et Jordanie. Outre les organisations « islamistes », un pays comme le Liban - ou la Syrie d'ailleurs - regorgent d'ONG étiquetées comme chrétiennes. Au sein de cette nébuleuse, les ONG occidentales peuvent éprouver des difficultés pour situer leurs champs d'action et surtout pour asseoir leur légitimité. Car les ONG locales les plus militantes peuvent entrer en concurrence, sur le terrain même de l'action, avec des ONG occidentales qui souffrent bien souvent, auprès des populations potentiellement bénéficiaires de l'aide, d'un véritable déficit de crédibilité en terme d'image; les grandes ONG internationales demeurent, dans certains contextes, perçues comme des organisations missionnaires, hostiles aux valeurs de l'Islam et instrumentées par les Etats occidentaux (cas de l'Irak, de l'Afghanistan).

5 Ainsi, dans cette région du monde, rien n'échappe au contexte politico-sécuritaire et socio-culturel. Il suffit de voir la manière dont au Soudan certains membres d'ONG occidentales furent au centre d'une campagne d'agressions pour comprendre que toute présence, toute action ne sont jamais neutres.

\section{Les enjeux des concentrations de populations déplacées et réfugiées}

\section{A. Concentrations et polarisations : quelles raisons ?}

6 Nous avons choisi d'illustrer à travers trois dynamiques, parfois mêlées, les conséquences de ces concentrations de populations déplacées. Ces trois dynamiques sont: les déplacements en lien avec des pratiques économiques, religieuses et culturelles (exode rural, pèlerinage, échanges matrimoniaux), les déplacements liés à des conflits et les déplacements liés à des accidents climatiques. Elles ont en commun la masse de population concernée et une polarisation des flux par les villes. 
7 Les travaux présentés dans ce numéro mettent en lumière tout un faisceau de raisons aboutissant à la concentration des populations qui se sont déplacées.

Les ONG sont directement responsables d'une forte polarisation autour de l'aide qu'elles apportent et concourent à accentuer la densification de certaines zones, en particulier au sein de certains camps dans lesquels elles interviennent en priorité, comme le montre l'exemple du Darfour traité par Philippe Quintin. En s'implantant dans les rares villes de la région, uniques lieux à être desservis par des moyens de transport modernes (avions), les acteurs de l'aide humanitaire favorisent la concentration humaine aux abords de ces centres urbains. A une échelle moindre qu'au Soudan, le même type de dynamique est observable pour les réfugiés urbains à Damas. La présence d'associations caritatives et d'institutions religieuses actives dans l'aide aux réfugiés favorise leur concentration dans certains quartiers. Cette action des acteurs qui distribuent l'aide humanitaire aux populations réfugiées favorise également leur regroupement selon des logiques communautaires.

De la même manière, les migrants internes installés dans les grandes villes du ProcheOrient se concentrent généralement par affinité communautaire - ethnique ou confessionnel - comme Fabrice Balanche le mentionne. Concernant les réfugiés irakiens à Damas, Cyril Roussel décrit également des concentrations de populations selon des critères religieux. Ce phénomène s'observe aussi chez des populations déplacées, comme l'indique Philippe Quintin pour le Soudan, où la tribu, le clan favorisent les regroupements. La communauté est avant tout un réseau de solidarité qui fournit entraide et protection. De plus, pour les communautés confessionnelles et surtout lorsqu'elles sont minoritaires, leur concentration en milieu urbain permet une meilleure protection de leur identité culturelle en garantissant la poursuite de leur pratiques sociales, culturelles et cultuelles.

10 Enfin, Marc Lavergne expose une dimension plus politique, voire machiavélique, de l'enjeu que représente la concentration de populations déplacées en ville. Il se demande si le but de la guerre au Darfour n'est pas un moyen d'intégrer de force les ruraux dans les villes pour mieux contrôler terres et populations. L'auteur invite à réfléchir, comme indiqué plus haut, sur l'omniprésence du pouvoir politique dans cette région du monde. Le cas de la Syrie, présenté par Fabrice Balanche, présente un autre pan du contrôle politique des populations migrantes installées à Damas. Par le laisser-faire en matière de constructions illégales, l'Etat s'assure d'une clientèle ou tout le moins construit un système d'interdépendance profond avec des populations qui lui sont redevables de la non-application du droit. Dans tout les cas, il semble évident que toutes ces populations déplacées n'ont pas vocation à rentrer dans leur territoire d'origine.

\section{B. Quand le provisoire se pérennise}

Les déplacements massifs de populations, qu'ils soient étalés sur une période longue exode rural - ou bien qu'ils soient intenses et violents comme lors de conflits par exemple, ont généralement comme corollaire l'émergence de zones de fortes densités (concentration de réfugiés dans certains quartiers urbains, camps péri-urbains, camps ruraux) dont le caractère temporaire disparaît avec le temps. En fonction des cas, les évolutions sont nombreuses. De déplacements de populations rurales au Darfour naissent des structures originales que l'on peut qualifier de préurbaines, comme l'explique Philippe Quintin. Les densités y sont élevées mais peu d'activités permettent 
de qualifier ces espaces précaires - pourtant appelés à perdurer - de quartiers urbains ou même périurbains. Néanmoins ces déplacés " aux portes des villes » apportent avec eux leur part d'évolutions tant sociales qu'économiques et se retrouvent bientôt confrontés brutalement à un véritable bouleversement de leur mode de vie, qu'ils le souhaitent ou non. Avec un conflit qui se prolonge dans le temps et le rôle actif des femmes, certains camps deviennent complémentaires des villes et évoluent vers des formes d'urbanisation primaires.

La situation décrite par Cyril Roussel illustre la diversité des situations. Dans les banlieues de Damas, les réfugiés urbains, originaires des villes irakiennes, s'insèrent dans le tissu urbain et contribuent à le modifier. Nous ne sommes pas ici dans une situation d'urgence face à des populations démunies, mais devant une catégorie de réfugiés urbains qui disposent généralement d'un savoir-faire, d'expériences et de réseaux relationnels, confrontée au problème de l'adaptation dans un pays qui n'est pas le leur et de la mise en place de stratégies de développement économique pour assurer leur survie dans l'exil. Dans cette diversité de contextes, les ONG se trouvent en prise avec des milieux et des situations en perpétuelle mutation. Habituellement aguerries aux conditions difficiles et à l'urgence, elles sont alors témoins d'une situation évolutive qui change de nature avec le temps et dont il faudrait revoir les approches et le traitement.

La pérennité d'une population allogène en un lieu pose la question de son insertion dans la société d'accueil. L'exemple des Palestiniens réfugiés au Liban, présenté par Daniel Meier, permet de saisir la complexité de l'intégration sociale d'une population en exil sur un temps long. A travers son objet d'étude, les mariages libano-palestiniens, il montre comment persistent les résistances à l'intégration qui marginalisent trop souvent les populations réfugiées. Cet article invite à réfléchir, au-delà du cas étudié, au devenir de toutes populations déplacées ou réfugiées dans un contexte de pérennité du conflit.

\section{Villes et banlieues en mutation}

Comme nous l'avons mentionné, l'implantation en milieu urbain constitue un dénominateur commun des populations déplacées et réfugiées au Proche-orient. Les points d'ancrage des migrants sont donc des espaces urbains ou péri-urbains dont les caractéristiques spatiales, sociales et économiques se modifient rapidement. De la formation de nouveaux territoires, à celle de nouvelles cultures, ou encore à celle de nouvelles communautés urbaines, les migrations vers les villes du Proche-Orient posent de nombreuses questions passionnantes. Observer, décrire et analyser ces flux de migrants vers les villes, nous paraissent les éléments d'une démarche essentielle à la compréhension de la production urbaine dans la région.

La production d'espaces urbains ne peut se comprendre, dans une zone de la planète touchée par des conflits à répétition et des mouvements massifs de population tant internes que transnationaux, sans une attention particulière portée aux banlieues des grandes agglomérations de la région. Les banlieues sont les principaux réceptacles de ces flux de migrants ou de réfugiés. Une des conséquences les plus probantes de l'arrivée de nouvelles populations dans les périphéries des grandes métropoles de cette partie du monde arabe demeure, selon nous, la mise en place d'un processus d'éclatement urbain, pour ne pas dire de ségrégation. Ce phénomène, que l'on peut 
définir comme le processus d'approfondissement des différenciations internes au sein des villes (Laborie, 1996), est au cœur de notre questionnement. Car le rôle que joue l'appartenance familiale, clanique, confessionnelle ou régionale dans la facilitation de l'implantation des nouveaux venus dans la ville semble mener à la formation, dans ses périphéries, de territoires urbains dans lesquels les styles de vie s'homogénéisent, les appartenances communautaires s'affirment. Outre Beyrouth et Bagdad, qui ont toutes deux subies des conflits inter-communautaires débouchant sur une homogénéisation confessionnelle de leurs quartiers, une ville comme Damas semble suivre une évolution proche dans sa structure socio-spatiale. Ici le processus ne s'appuie pas sur la violence armée mais sur une dynamique culturelle et religieuse qui remet en cause bien des principes issus de l'idéologie socialiste laïque arabe. Loin de prophétiser un scénario à l'irakienne dans les autres villes de la région, il n'en demeure pas moins que l'analyse socio-spatiale est révélatrice de dynamiques sectaires, avec tous les risques de dérives que cela comporte.

\section{BIBLIOGRAPHIE}

Chatelard G., Doraï M. K., 2009, « La présence irakienne en Syrie et en Jordanie : dynamiques sociales et spatiales, et modes de gestion par les pays d'accueil », Maghreb-Machrek, n¹99, p. 43-60.

Dato J, et al., 2008, « Comment intervenir au Proche et Moyen-Orient », Humanitaire, $\mathrm{n}^{\circ} 20, \mathrm{p}$. 14-27.

Laborie J.-P., 2006, « La ville face à ses territoires », Courrier du CNRS, nº 82 « Villes, Cities, Ciudades ».

\section{AUTEUR}

\section{ROUSSEL CYRIL}

Géographe, chercheur associé au Gremmo de Lyon - UMR 5195 GREMMO

cyril.roussel@netcourrier.com 